\title{
Psicopatia e Autores de Violência Sexual contra Crianças e Adolescentes
}

\author{
Julia Nunes de Souza Teixeira ${ }^{1}$ (D) \\ Pontifícia Universidade Católica de Goiás, Goiás-GO, Brasil \\ Universidade do Estado do Rio de Janeiro, Rio de Janeiro-RJ, Brasil \\ Ana Cristina Resende (i) \\ Pontifícia Universidade Católica de Goiás, Goiás-GO, Brasil \\ Rodrigo Perissinotto (D) \\ Pontifícia Universidade Católica de Goiás, Goiás-GO, Brasil \\ Centro Universitário de Mineiros, Mineiros-GO, Brasil
}

\section{RESUMO}

O objetivo do presente estudo foi avaliar diferenças no perfil criminal de autores de violência sexual contra crianças e adolescentes (AVS) considerados psicopatas e não psicopatas. Participaram 30 reeducandos cumprindo pena em regime fechado, que foram divididos em dois grupos: G1, composto pelos AVS considerados psicopatas (PCL-R $\geq 30$ ); e G2, composto pelos AVS considerados não psicopatas (PCL-R <30). Para a coleta de dados, foi utilizado o Psychopathy Checklist - Revised (PCL-R), e as análises foram feitas por meio de estatísticas descritiva e comparativa. Os resultados apontaram diferenças significativas entre os grupos com relação ao tempo de pena, à quantidade de fuga e rebelião, assim como o número de processos e de vítimas, mostrando que os AVS psicopatas são mais indisciplinados e têm mais chances de reincidir criminalmente do que os não psicopatas, e que, comumente, costumam vitimizar mais pessoas e cometer uma maior variedade de crimes do que os não psicopatas.

Palavras-chave: personalidade antissocial; violência sexual; psicologia criminal.

\section{ABSTRACT - Psychopathy and Sex Offences against Children and Adolescents}

The aim of the present study was to evaluate differences in the profiles of sex offenders considered psychopaths and nonpsychopaths that committed crimes against children and adolescents. The subjects of this study were 30 re-educated criminals serving a prison sentence, organized into two groups: G1, consisting of sex offenders considered psychopaths (PCL-R $\geq 30$ ); and G2, consisting of non-psychopaths (PCL-R $<30)$. The Psychopathy Checklist-Revised (PCL-R) was used to collect data, and the analyses were performed using descriptive and comparative statistics. The results presented significant differences between the two groups in terms of the amount of time spent in incarceration, the quantity of prison breaks and rebellions, and the number of cases and victims. This shows that psychopathic sex offenders are more undisciplined and more recidivists than non-psychopaths, and that they often victimize more people and commit a greater variety of crimes than non-psychopaths.

Keywords: antisocial personality; sexual violence; criminal psychology.

\section{RESUMEN - Psicopatía en los Autores de Violencia Sexual Contra Niños y Adolescentes}

El objetivo del presente estudio fue evaluar diferencias en el perfil criminal de los autores de violencia sexual contra niños y adolescentes (AVS) considerados psicópatas y no psicópatas. Participaron 30 reclusos con sentencia de régimen cerrado, organizados en dos equipos. G1, compuesto por los AVS considerados psicópatas (PCL-R $\geq 30$ ); y G2, formado por los AVS, considerados no psicópatas (PCL-R<30). Para la recopilación de datos, se utilizó el Psychopathy Checklist-Revised (PCL-R), y los análisis se realizaron utilizando estadísticas descriptivas y comparativas. Los resultados señalaron distinciones significativas entre los grupos con relación al tiempo de condena, la cantidad de fugas y rebeliones; así como el número de demandas y de víctimas; demostrando que los AVS psicópatas son más indisciplinados y tienen más probabilidades de reincidir al crimen, además de victimizar a más personas y cometer a una mayor variedad de delitos que los no psicópatas.

Palabras clave: personalidad antisocial; violencia sexual, psicología criminal.

De acordo com a literatura, a maior parte dos crimes violentos é cometida por pessoas com psicopatia (Hare, 2006; Krstic et. al., 2017; Neumann \& Hare, 2008). A psicopatia é considerada um transtorno de personalidade de difícil remissão, e pode ser diferenciada dos demais transtornos por seus padrões característicos de sintomas interpessoais, afetivos e comportamentais. Considerando os sintomas interpessoais, os psicopatas se destacam por serem egocêntricos, manipuladores, dominadores e por estabelecerem vínculos mais superficiais e instáveis. Em relação aos sintomas afetivos, os psicopatas revelam superficialidade, frieza, falta de empatia, falta de remorso ou culpa e falta de ansiedade. No que diz respeito aos sintomas comportamentais, eles violam regras sociais,

${ }^{1}$ Endereço para correspondência: Rua André Cavalcanti, 95, apto 202, Centro, 20231-050, Rio de Janeiro, RJ. Tel.: (21) 99860-9745. E-mail: julia84.psi@gmail.com Artigo derivado da 'Dissertação de mestrado' de 'Julia Nunes de Souza Teixeira' com orientação de 'Ana Cristina Resende', defendida em '2017' no programa de pós-graduação ‘Stricto Sensu em Psicologia’ da 'Pontifícia Universidade Católica de Goiás. 
são impulsivos e irresponsáveis, ávidos de sensações e mais predispostos a fazer uso de substâncias psicoativas (Hare \& Neumann, 2006; Hill et al., 2008; Verschuere et al., 2017).

Segundo Frick e Marsee (2018), a vida criminosa dos psicopatas tem início precoce, geralmente na adolescência. No sistema prisional, são os mais indisciplinados, apresentando respostas não satisfatórias nos programas de reabilitação e os com elevado índice de reincidência criminal (Balsis et al., 2017). De acordo com Hare (1995), os psicopatas representam, em média, $1 \%$ da população geral e $15 \%$ a $20 \%$ da população carcerária. Quando se trata de Autores de Violência Sexual (AVSs), essa porcentagem pode ser ligeiramente maior (Hare, 2003; Hauck Filho et al., 2012; Hare et al., 1991; Hemphill et al., 1998). A taxa de reincidência entre os AVSs varia entre $18 \%$ e $45 \%$. Hill et al. (2008) reiteram que, quanto mais violento for o crime cometido, maior é a chance de que o criminoso volte a repeti-lo.

Uma operacionalização do construto psicopatia, ou um diagnóstico desse transtorno, pode ser realizada por meio do Psychopathy Checklist - Revised (PCL-R) (Hare, 2003), uma escala que inicialmente era composta por dois fatores. $O$ fator 1 é definido pelas características nucleares dos traços da personalidade que compõem o perfil prototípico da condição de psicopatia, incluindo as facetas afetivas (falta de remorso e culpa, insensibilidade afetiva, falta de empatia ou maldade) e interpessoal (charme superficial, grandiosidade, manipulação. superficialidade, falsidade, insensibilidade/crueldade). $\mathrm{O}$ fator 2, por sua vez, é definido por comportamentos mais típicos do Transtorno de Personalidade Antissocial, que tende a se desenvolver em pessoas que vivem em contextos problemáticos, tais como viver em uma família desestruturada ou em bairros tomados pelo tráfico de drogas. Esse segundo fator seria composto por duas facetas: estilo de vida parasitária (não necessariamente é comportamento criminoso) e faceta antissocial (consistente com os comportamentos criminosos comuns na população carcerária, impulsividade e irresponsabilidade).

Contudo, uma década após a publicação do PCL-R, Cooke e Michie (2001) não conseguiram replicar a validade estrutural do modelo de 2 fatores. Desse modo, os autores encontraram um modelo de três fatores ao analisar os mesmos itens do instrumento de dois fatores: afetivo, interpessoal e comportamental (descartando cinco itens que mediam criminalidade). Então, a partir de novos estudos, Hare (2003) propôs um modelo de quatro fatores, incluindo os três fatores anteriormente citados e o fator 4, que é a criminalidade (composto pelos cinco itens que tinham sido retirados para compor a escala de três fatores). Assim, o mesmo instrumento de dois fatores pode ser agora analisado a partir de quatro fatores, mantendo os itens da versão original de dois fatores.

O PCL-R tem sido uma ferramenta de suporte válida para auxiliar na tomada de decisões no meio forense em diferentes países, especialmente nos Estados Unidos, quando é necessário considerar a capacidade de resposta a tratamentos psicoterapêuticos (D'Silva et al., 2004), a probabilidade de reincidência de crimes comuns, reincidência de comportamentos violentos e reincidência de abuso sexual (Hawes et al., 2013; Mokros et al., 2014; Olver \& Wong, 2009). Além disso, tem sido útil para categorizar os AVSs e compará-los com detentos que cometeram outros tipos de crimes (Singh et al., 2011; Verschuere et al., 2017). O instrumento também tem sido usado principalmente em contextos de alto risco, tais como determinações de liberdade condicional, pena de morte e a capacidade de um AVS assumir um compromisso civil ou ter uma progressão de pena (Blais, 2015; DeMatteo et al., 2014; Guy et al., 2015).

Neste estudo, o termo AVS é utilizado para se referir às pessoas do sexo masculino que cometeram qualquer tipo de agressão sexual. Os AVSs compõem um grupo heterogêneo, provenientes de backgrounds diferentes, com uma significativa variedade no tipo e no modo de cometerem seus crimes (Huss, 2011; Soares et al., 2016).

No Brasil, o perfil mais comum do AVS contra crianças e adolescentes, destacado em outros estudos, é um agressor homem, com idade entre 30 e 40 anos, em sua maioria de etnia parda, com ensino fundamental incompleto, solteiro e trabalhador braçal (Soares et al., 2016). Frequentemente, esse homem responde criminalmente apenas a processos sexuais, não possui histórico de reincidência criminal, é próximo de sua vítima (pai, padrasto, tio ou vizinhos, por exemplo), tem preferência por vítimas crianças e adolescentes e, geralmente, escolhe como local do abuso a residência da vítima ou a sua própria (Martins \& Jorge, 2010; Santos et al., 2015; Serafim et al., 2009; Soares et al. 2016).

De acordo com o perfil de personalidade, alguns autores têm classificado os AVSs de crianças e adolescentes em quatro categorias: pedófilos, psicóticos ou com retardo mental, AVS situacional, psicopatas ou antissociais. Para Sharma (2003), a pedofilia é um transtorno parafílico que envolve fantasias recorrentes, intensas e sexualmente excitantes com crianças ou pré-púberes, assim como pode envolver impulsos sexuais ou comportamentos envolvendo atividade sexual com essas vítimas bem jovens. Os AVSs pedófilos podem ser abusadores ou molestadores. Os abusadores geralmente são mais sutis e discretos, tendem a ser indivíduos imaturos e com poucas habilidades sociais e, comumente, envolvem-se com material pornográfico infantil. Os molestadores têm um padrão de comportamento mais invasivo e agressivo. Podem ser mais inteligentes que os abusadores e agem de forma premeditada. Sabem que estão agindo fora da lei, porém, intelectualizam seu comportamento, convencendo-se de que não estão cometendo nenhum crime e que seu comportamento é socialmente aceitável (Huss, 2011; Misaka, 2014; Serafim et al., 2009). 
Com relação aos AVSs psicóticos ou com retardo mental, são indivíduos com maior dificuldade para discernir quais são os comportamentos mais adequados para várias situações interpessoais ou sociais, bem como de entender quais são as consequências de seus ato com relação ao seu comportamento sexual. Não são capazes de perceber o caráter delituoso dos atos sexuais que praticam e, no geral, não manifestam um comportamento agressivo para com a vítima ( $\mathrm{Lu}$ et al., 2015; Serafim et al., 2009).

Os AVS situacionais tendem a ser os mais oportunistas e impulsivos, avaliam as características gerais da vítima e usam como critérios de escolha a disponibilidade da vítima e a oportunidade apresentada. Podem ser pessoas que foram expostas a situações de estresse extremo. Geralmente, são pessoas que possuem poder absoluto sobre a vítima e têm dificuldade no controle dos impulsos (Holmes \& Holmes, 2002; Serafim et al., 2009).

Os AVSs psicopatas e antissociais são indivíduos com baixa frequência afetiva, com reduzida ou nenhuma posição empática, apresentando comportamentos impulsivos e comumente se envolvem com criminalidade e abuso de substâncias psicoativas, com condutas sexuais de maior crueldade. Sentem-se satisfeitos com o sofrimento da vítima, esta sendo reduzida a um objeto de degradação e rejeição (Gacono et al., 2008; Hare, 2006; Hill et al., 2008; Morana, 2003; Krstic et al., 2017).

No que diz respeito ao diagnóstico de AVSs como psicopatas por meio do PCL-R, Riquelme et al. (2004) fizeram um estudo com 60 AVSs condenados pelos seus crimes em San Miguel (Chile). Aqueles AVSs considerados psicopatas (PCL-R>31) apresentavam maior probabilidade de reincidir criminalmente e tendiam a reincidir com maior frequência. Os resultados apontaram que esses AVSs possuíam mais características interpessoais e emocionais que são próprias da psicopatia, do que características de estilo de vida e comportamento antissocial, e tiveram pontuações significativamente maiores no PCL-R do que indivíduos condenados por outros tipos de crimes.

O estudo de Valverde (2005) corrobora com a investigação de Riquelme et al. (2004). Os autores concluíram que há uma relação positiva entre risco de violência sexual e a psicopatia. Ou seja, AVSs psicopatas apresentavam maior probabilidade de reincidência no crime sexual, bem como cometem, significativamente, mais crimes violentos do que os não psicopatas. O coeficiente de correlação bisserial entre reincidência e traços de psicopatia, no estudo de Riquelme et al. (2004), foi de 0,308. No estudo de Valverde (2005), não é apresentado o coeficiente de correlação entre psicopatia e reincidência, apenas citado que há uma relação positiva entre as duas instâncias. No estudo, os resultados apontaram que 35\% dos AVSs preenchiam os critérios para psicopatia (PCL-R $>30$ ). Os AVS não psicopatas foram classificados como abusadores oportunistas (16,7\%), sádicos sexuais (15\%) e abusadores vingativos (13\%). Os demais não se enquadravam em nenhuma das categorias.

Em um estudo mais recente, realizado por Krstic et al. (2017), teve como objetivo examinar traços psicopáticos em uma amostra de 958 AVSs. Destes, 561 foram avaliados no Centro de Tratamento de Massachusetts (MTC) para pessoas sexualmente perigosas. Os outros 397 infratores do MTC eram de vários centros de tratamento ambulatorial e instituições forenses em Minnesota, Nova Jersey e Massachusetts. A amostra foi dividida em quatro subtipos: psicopatia prototípica, conicidade insensível, sociopatia e criminosos gerais. Os fatores que avaliam a área afetiva e o comportamento antissocial foram preditores de crimes sexuais violentos ( $p$ 's $<0,001)$. Os resultados mostraram que os AVSs com maior número de traços de psicopatia prototípica (pontuação alta nos quatro fatores da escala e PCL-R total $>30$ ) cometeram crimes sexuais mais violentos do que os demais AVSs.

Desse modo, tomando como base o que foi exposto, o presente estudo teve como objetivo geral avaliar diferenças no perfil criminal de AVSs contra crianças e adolescentes considerados psicopatas e não psicopatas. Os objetivos específicos foram: descrever o perfil dos AVSs considerados psicopatas (G1) e não psicopatas (G2); verificar se existem diferenças entre os grupos (G1 e G2) quanto à presença de traços psicopáticos por meio dos fatores do PCL-R; avaliar a diferença entre esses dois grupos (G1 e G2) com relação às variáveis sociodemográficas (idade, escolaridade, estado marital, raça, filhos, número de filhos e profissão) e do perfil criminal (número de processos, número de processos por violência sexual, tipo de crime, número de vítimas, sexo das vítimas, proximidade com a vítima e local do crime).

No Brasil, poucos estudos foram realizados utilizando o PCL-R para coleta de dados, e nenhum estudo avalia diferenças no perfil criminal de autores de violência sexual contra crianças e adolescentes com traços de psicopatia. Trata-se de um estudo inédito no contexto brasileiro, trazendo dados consistentes e discussões ricas à comunidade científica. Aprofundar a investigação sobre o tema psicopatia no Brasil, em uma população carcerária, pode ajudar a compreender algumas nuances do comportamento criminal e contribuir à elaboração de estratégias que auxiliem na diminuição do comportamento criminal. Assim, as seguintes hipóteses foram levantadas:

- AVSs com mais traços de psicopatia apresentam um perfil criminal mais violento;

- AVSs com mais traços de psicopatia apresentam um perfil social (idade, escolaridade) diferente dos AVSs não psicopatas;

- AVSs com mais traços de psicopatia têm mais chances de reincidir criminalmente, quando comparados a AVSs não psicopatas. 


\section{Método}

\section{Delineamento do Estudo}

Trata-se de um estudo descritivo, comparativo, de campo, que busca conhecer e interpretar a realidade estudada, fazendo uso de métodos padronizados e observação naturalista para coleta de dados.

\section{Participantes}

Foram convidados a participar do estudo 31 reeducandos, sendo que um participante se recusou a fornecer informações, sendo excluído da pesquisa. Desse modo, participaram do presente estudo 30 reeducandos AVSs contra crianças e adolescentes, com idade entre 18 e 60 anos. Os critérios de inclusão dos participantes da pesquisa foram: 1 . reeducandos do sexo masculino condenados por crimes sexuais; 2 . cumprindo pena em regime fechado; 3. ter vitimado crianças e/ou adolescentes. Os critérios de exclusão dos participantes foram: 1. reeducandos que não forneceram informações suficientes durante a entrevista; 2 . reeducandos cujos prontuários do cartório não ofertaram os dados necessários e relevantes à pesquisa.

Os 30 participantes foram divididos em dois grupos de estudo: o Grupo 1 (G1) e o Grupo 2 (G2). O G1 foi constituído por dez AVSs com maior número de traços de psicopatia, ou seja, com pontuação maior ou igual a 30 no PCL-R. O G2 foi constituído pelos demais AVSs que tiveram pontuações menores do que 30 no PCL-R.

$\mathrm{O}$ ponto de corte utilizado neste estudo seguiu o mesmo utilizado em outros estudos internacionais voltados para discriminar pessoas com e sem psicopatia, numa perspectiva categorial (Krstic et al., 2017; Young et al., 2010). No Brasil, não há estudos que fundamentem esse ponto de corte, mesmo porque Morana (2004), que validou a escala para o Brasil, não tinha o interesse na identificação da psicopatia, uma vez que não há leis diferenciadas para pessoas que cometem crimes e possuem esse diagnóstico em território brasileiro. A autora preocupou-se em estabelecer um ponto de corte para identificar criminosos com maior ou menor potencial de reincidência criminal. As informações sobre as vítimas dos participantes foram coletadas por meio dos dados contidos nos prontuários disponíveis no cartório da unidade prisional.

\section{Instrumento}

Psychopathy Checklist - Revised (PCL-R). Desenvolvida e validada por Rorbert Hare (Hare, 1991, 2003) para avaliar a psicopatia em populações forenses masculinas. No Brasil, foi validada por Morana (2004). Esse instrumento é composto por três partes: um roteiro de entrevista semiestruturada; um roteiro para investigação de dados objetivos e de informações adicionais que, no caso do presente estudo, foi preenchido somente com informações provenientes do processo criminal; uma escala numérica ordinal de três pontos $(0,1$ ou 2$)$ de 20 itens, que qualifica os reeducandos de acordo com o grau em que cada comportamento dele condiz com a descrição do item. A escala deve ser preenchida por pessoas treinadas para respondê-la e que já têm conhecimento prévio das informações da entrevista e do roteiro com dados objetivos e adicionais sobre o reeducando.

A escala é dividida em dois fatores: o Fator 1, com oito itens relacionados à traços mais frequentes em pessoas com psicopatia; e o Fator 2, com nove itens, geralmente mais frequentes em pessoas com Transtorno de Personalidade Antissocial (TPA). Além desses dois fatores há três itens na escala que não se enquadraram estatisticamente em nenhum dos fatores - comportamento sexual promíscuo; muitas relações conjugais de longo prazo; versatilidade criminal - porém, são usados para derivar um escore total no PCL-R (Morana, 2004).

\section{Procedimentos}

Inicialmente, o projeto foi aprovado pela direção da penitenciária e submetido e aprovado por um Comitê de Ética em Pesquisa (CEP). Em seguida, foi realizada a coleta de dados nos prontuários no cartório da penitenciária de 30 reeducandos escolhidos aleatoriamente. Aqueles que aceitaram, assinaram o Termo de Consentimento Livre e Esclarecido (TCLE) e, logo em seguida, foi realizada a entrevista. O tempo para coletar os dados de cada participante foi de aproximadamente três horas: uma hora para levantar as informações no processo, em que foram coletadas informações sobre o crime, sobre as vítimas dos participantes e sobre adicionais disponíveis no cartório da unidade prisional; duas para a entrevista do PCL-R; e, aproximadamente, quinze minutos para preencher a escala de 20 itens.

Posteriormente, $60 \%$ dos protocolos do PCL-R $(N=20)$ foram classificados aleatoriamente e corrigidos por dois juízes, para o cálculo da análise de concordância entre os avaliadores, por meio do Índice de Concordância Intraclasse/Intraclass Correlation Coefficient (ICC). Os valores de referência para o ICC foram: entre 0,40 e 0,59 considerou-se os valores moderados, entre 0,60 e 0,74 bons e acima de 0,74 excelentes (Cicchetti, 1994; Hunsley \& Mash, 2008; Shrout \& Fleiss, 1979). O ICC médio foi de 0,89 (variando entre 0,62 e 0,96), e a mediana 0,91 . Outra análise de fidedignidade foi realizada por meio do coeficiente alfa de Cronbach. Para o Fator 1, o coeficiente alfa foi 0,79 , e para o F2 foi 0,94 . No geral, a consistência interna do total de pontuações com o PCL-R pode ser considerada aceitável para uma escala clínica, com índice de 0,93 para todos os itens. Esses valores são semelhantes àqueles alcançados no estudo brasileiro do PCL-R realizado por Morana (2003).

Após as análises de fidedignidade do PCL-R, seguiu-se com os levantamentos de pontos alcançados em todos os protocolos do PCL-R, e as análises foram 
realizadas por meio de estatísticas descritivas e comparativas utilizando o Statistical Package for the Social Sciences (SPSS). As variáveis qualitativas foram apresentadas como frequência absoluta e relativa, e as quantitativas como média e desvio padrão $(D P)$. Verificou-se a diferença estatística entre os grupos de participantes (G1 versus G2). Para as variáveis qualitativas, foi realizado o teste de qui-quadrado de Pearson para tendência ou exato de Fisher e, para as quantitativas, o teste $t$ de Student para amostras independentes. Em todas as análises, valores de $p<0,05$ foram considerados estatisticamente significativos. Além disso, foram verificados os tamanhos dos efeitos entre os grupos e algumas variáveis, utilizando o $d$ de Cohen (1988) para as variáveis quantitativas e o $v$ de Cramer para as qualitativas. O tamanho do efeito será classificado como pequeno $(d=0,20$ a 0,49$)$, médio $(d=0,50$ a 0,79$)$ e grande $(d>0,80)$, com base nos valores de referência sugeridos por Cohen (1988). Para o $v$ de Cramer, o efeito será classificado como pequeno $(v=0,10$ a 0,20$)$, médio $(v=0,21$ a 0,60$)$ e grande $(v=0,61$ a 1,0$)$, usando como referência os valores sugeridos por Rea e Parker (1992).

\section{Resultados}

Considerando o perfil sociodemográfico dos participantes, observou-se que a idade variou entre 18 e 60 anos, com uma média de 33,5 anos $(D P=9,2)$. No que diz respeito à escolaridade, a maioria dos participantes $(N=19,63,3 \%)$ possuía de cinco a oito anos de estudo. Quanto à raça/cor da pele, 43,3\% $(N=13)$ dos participantes se autodeclararam brancos, seguidos de $30 \%(N=9)$ de raça parda e $13,3 \%(N=4)$ da raça negra. Com relação ao estado marital, $40 \%(N=12)$ eram casados. Do total de participantes, $46,7 \%(N=14)$ possuíam filhos, sendo a média de filhos de 2,9 ( $D P=2,2$; mínimo: 1, máximo: 9). A profissão predominante foi o trabalho braçal, especialmente da construção civil com $40,0 \%(N=12)$, seguido de manutenção/serviços gerais com 16,7\% $(N=5)$ dos participantes, vigilante/motorista com 13,3\% $(N=4)$, autônomos com $10 \%(N=3)$ e trabalhadores rurais com $6,7 \%(N=2)$.

Quanto ao perfil criminal, foram levantados os seguintes aspectos: a idade das vítimas (se crianças ou adolescentes são vitimizados), o número de vítimas, o sexo delas, a proximidade dos agressores, versatilidade criminal, local mais frequente em que o crime foi cometido e o número de processos respondidos (processos totais e por crimes sexuais).

No que se refere à idade das vítimas, a média foi de 10,0 anos ( $D P=2,9$; mínimo: 5; máximo: 15$)$. Quanto ao número de vítimas, a média foi de 1,6 $(D P=1,1$; mínimo: 1; máximo: 6). Com relação ao sexo da vítima, $86,7 \%(N=26)$ eram do sexo feminino.

Com relação à proximidade do agressor com a vítima, 66,7\% $(N=20)$ dos participantes tinham algum tipo de proximidade (pai, padrasto ou tio), sendo que desses, $85 \%(n=17)$ eram pais ou padrastos. Quanto ao local do crime, $50 \%(N=15)$ dos abusos ocorreram na residência da vítima, $26,7 \%(N=8)$ na residência do AVS, $10 \%$ $(N=3)$ em terreno baldio ou matagal e $13,3 \%(N=4)$ em outros locais não especificado.

Quanto aos processos criminais, 76,7\% $(N=23)$ dos participantes respondiam a um processo e $23,3 \%(N=7)$ entre dois e cinco processos criminais. Quanto à versatilidade criminal, a maioria dos participantes $(76,7 \%$, $N=23$ ) apresentou somente crimes sexuais. Com relação à reincidência, o dado não pôde ser mensurado estatisticamente devido à ausência de dados referentes a essa variável, tanto no processo como na entrevista. Os dados sobre reincidência foram coletados nos prontuários do cartório da penitenciária, porém, nem todos continham informações sobre reincidência, em que as que apareceram foram contabilizadas, mas não usadas para inferir resultados.

No que diz respeito aos escores dos fatores no PCL-R, $73,3 \%$ da amostra $(N=22)$ tiveram pontuações mais elevadas no Fator 1 (traços de psicopatia), e $26,7 \%(N=8)$ apresentaram pontuações mais altas no Fator 2 (traços antissociais). Considerando os fatores (F1 e F2) em cada Grupo (G1 e G2), observou-se que os participantes do G1 (considerados psicopatas) obtiveram frequências significativamente maiores, tanto no F1 quanto no F2, quando comparados aos fatores do G2. O G2 (considerados criminosos comuns) obteve índices mais elevados de F1 do que F2, o que não seria o mais óbvio, uma vez que não preenchem os critérios para psicopatia.

A Tabela 1 apresenta a estatística descritiva e comparativa dos grupos (G1, G2), além dos resultados alcançados no grupo com um todo (GT), com relação à pontuação nos Fatores 1 e 2 e a pontuação total no PCL-R.

A Tabela 2 apresenta a estatística descritiva e comparativa das variáveis quantitativas (idade ao ser preso, número médio de vítimas, processos, processos por violência sexual, reincidência, fuga, rebelião, pena em anos, quantidade de vítimas crianças, vítimas adolescente e vítimas adultos) com relação ao grupo total (GT) e as diferenças entre os grupos. O G1 apresentou média de idade $(M=28,4 ; D P=7,6)$ significativamente menor $(p=0,029)$ que o $\mathrm{G} 2(M=36,1 ; D P=9,2)$. Contudo, o G1 apresentou médias significantemente maiores de número de vítimas $(p=0,006)$, fugas $(p=0,009)$, rebeliões $(p=0,014)$, tempo total de pena $(p=0,001)$, vítima adolescente e adultas $(p=0,024)$. O tamanho do efeito ( $d$ de Cohen) foi grande para essas variáveis, mudando de 0,70 a 1,21 .

Não houve diferenças significantes entre os grupos com relação à vítima criança $(p=0,797)$, em que o tamanho do efeito também foi muito pequeno ( $d$ de Cohen $=-0,200$ ). 
Tabela 1

Pontuação por Fator e Pontuação Total no PCL-R para G1, G2 e GT

\begin{tabular}{|c|c|c|c|c|c|c|c|c|}
\hline & & $M$ & $\mathrm{DP}$ & Min & Max & $t$ & $p$ & $d$ \\
\hline \multirow{3}{*}{ Fator 1} & G1 & 14,3 & 1,6 & 11,0 & 16,0 & \multirow{3}{*}{2,45} & \multirow{3}{*}{0,021} & \multirow{3}{*}{1,052} \\
\hline & G2 & 11,9 & 2,8 & 6,0 & 16,0 & & & \\
\hline & GT & 12,7 & 2,7 & 6,0 & 16,0 & & & \\
\hline \multirow{3}{*}{ Fator 2} & G1 & 14,5 & 2,6 & 11,3 & 18,0 & \multirow{3}{*}{5,26} & \multirow{3}{*}{$<0,001$} & \multirow{3}{*}{2,165} \\
\hline & G2 & 7,7 & 3,6 & 1,3 & 12,9 & & & \\
\hline & GT & 10,0 & 4,6 & 1,3 & 18,0 & & & \\
\hline \multirow{3}{*}{ PCL-R } & G1 & 33,7 & 4,2 & 30,0 & 40,0 & \multirow{3}{*}{5,95} & \multirow{3}{*}{$<0,001$} & \multirow{3}{*}{0,312} \\
\hline & G2 & 22,6 & 2,1 & 11,1 & 29,0 & & & \\
\hline & GT & 26,3 & 7,1 & 11,1 & 40,0 & & & \\
\hline
\end{tabular}

Nota. G1=indivíduos com PCL-R $\geq 30$ (N=10); G2=indivíduos com PCL-R<30 (N=20); GT=total da amostra (N=30)

Tabela 2

Diferenças entre Grupos para Variáveis do Perfil Criminal

\begin{tabular}{|c|c|c|c|c|c|c|c|c|}
\hline & & $M$ & $D P$ & Min & Max & $t$ & $p$ & $d$ \\
\hline \multirow{3}{*}{ Idade ao ser preso } & G1 & 28,4 & 7,6 & 18 & 44 & \multirow{3}{*}{$-2,3$} & \multirow{3}{*}{0,029} & \multirow{3}{*}{0,918} \\
\hline & G2 & 36,1 & 9,1 & 23 & 60 & & & \\
\hline & GT & 33,5 & 9,2 & 18 & 60 & & & \\
\hline \multirow{3}{*}{$\mathrm{N}^{\circ}$ de vítimas } & G1 & 2,3 & 1,6 & 1 & 6 & \multirow{3}{*}{2,98} & \multirow{3}{*}{0,006} & \multirow{3}{*}{0,943} \\
\hline & $\mathrm{G} 2$ & 1,2 & 0,4 & 1 & 2 & & & \\
\hline & GT & 1,6 & 1,1 & 1 & 6 & & & \\
\hline \multirow{3}{*}{ Processos } & G1 & 2,1 & 1,5 & 1 & 5 & \multirow{3}{*}{1,9} & \multirow{3}{*}{0,068} & \multirow{3}{*}{0,665} \\
\hline & G2 & 1,3 & 0,8 & 1 & 4 & & & \\
\hline & GT & 1,6 & 1,1 & 1 & 5 & & & \\
\hline \multirow{3}{*}{ Processos por violência sexual } & G1 & 1,6 & 1,3 & 1 & 5 & \multirow{3}{*}{1,6} & \multirow{3}{*}{0,121} & \multirow{3}{*}{0,529} \\
\hline & G2 & 1,1 & 0,3 & 1 & 2 & & & \\
\hline & GT & 1,3 & 0,8 & 1 & 5 & & & \\
\hline \multirow{3}{*}{ Reincidência } & G1 & 0,7 & 0,5 & 0 & 1 & \multirow{3}{*}{1,75} & \multirow{3}{*}{0,074} & \multirow{3}{*}{0,799} \\
\hline & G2 & 0,3 & 0,5 & 0 & 1 & & & \\
\hline & GT & 0,5 & 0,5 & 0 & 1 & & & \\
\hline \multirow{3}{*}{ Fuga } & G1 & 1,1 & 0,9 & 0 & 3 & \multirow{3}{*}{2,79} & \multirow{3}{*}{0,009} & \multirow{3}{*}{0,961} \\
\hline & G2 & 0,4 & 0,5 & 0 & 1 & & & \\
\hline & GT & 0,6 & 0,7 & 0 & 3 & & & \\
\hline \multirow{3}{*}{ Rebelião } & G1 & 0,5 & 0,5 & 0 & 1 & \multirow{3}{*}{2,63} & \multirow{3}{*}{0,014} & \multirow{3}{*}{0,97} \\
\hline & G2 & 0,1 & 0,3 & 0 & 1 & & & \\
\hline & GT & 0,2 & 0,4 & 0 & 1 & & & \\
\hline \multirow{3}{*}{ Pena (anos) } & G1 & 34,1 & 23,4 & 8,6 & 92 & 352 & ( & ק० \\
\hline & G2 & 14,2 & 7,1 & 6,5 & 37,6 & 3,52 & 0,001 & 1,208 \\
\hline & $\mathrm{GT}$ & 20,8 & 17,1 & 6,5 & 92 & & & \\
\hline & G1 & 1 & 0,5 & 0 & 2 & 25 & 797 & - ? \\
\hline Vítimas crianças & G2 & 1,1 & 0,5 & 0 & 2 & $-U, \angle J$ & 0,191 & $-0,2$ \\
\hline & GT & 1 & 0,5 & 0 & 2 & & & \\
\hline & G1 & 0,6 & 0,8 & 0 & 2 & 28 & 024 & 827 \\
\hline Vítimas adolescentes & G2 & 0,1 & 0,3 & 0 & 1 & 2,30 & $0,0<4$ & $0,0<1$ \\
\hline & $\mathrm{GT}$ & 0,3 & 0,6 & 0 & 2 & & & \\
\hline
\end{tabular}


Tabela 2 (continuação)

Diferenças entre Grupos para Variáveis do Perfil Criminal

\begin{tabular}{lllllllll}
\hline & & $M$ & $D P$ & Min & Max & $t$ & $p$ & $d$ \\
\hline \multirow{3}{*}{ Vítimas adultos } & G1 & 0,9 & 1,6 & 0 & 5 & 2,37 & 0,024 & 0,701 \\
& G2 & 0,1 & 0,2 & 0 & 5 & & \\
\hline
\end{tabular}

Nota. G1=indivíduos com PCL-R $\geq 30$ (N=10); G2=indivíduos com PCL-R<30 (N=20); GT: total da amostra ( $N=30)$

Ainda em relação à idade com que os participantes foram presos, fez-se um estudo de correlação dessa variável com os fatores (F1 e F2) e escore total do PCL-R. Em relação ao $\mathrm{G} 1$, não se observou correlações significativas. Já em G2 houve correlação negativa entre idade e o Fator $2(r=-0,506 ; p=0,023)$. Em relação ao $\mathrm{GT}$, os resultados indicaram correlação negativa significativa entre idade e o Fator $2(r=-0,567 ; p=0,001)$ e o escore total do PCL-R $(r=-0,461 ; p=0,01)$. Isso sugere que quanto mais jovem era o AVS contra crianças e adolescentes maior era a tendência ao descontrole comportamental, à ausência de metas realistas e à impulsividade, traços geralmente mais frequente em pessoas com Transtorno de Personalidade Antissocial (TPA).

A Tabela 3 mostra a relação entre a idade com que os participantes foram presos de cada grupo (G1 e G2) e do grupo total (GT), e os fatores (F1 e F2) e escore total do PCL-R. Os resultados referentes ao GT indicaram uma correlação negativa significativa entre idade e o Fator 2 e o escore total do PCL-R. Isso sugere que quanto mais jovem é o AVS contra crianças e adolescentes, maior era a tendência ao descontrole comportamental, à ausência de metas realistas e à impulsividade, traços geralmente mais frequente em pessoas com Transtorno de Personalidade Antissocial (TPA).

A Tabela 4 apresenta a estatística descritiva com relação ao GT e aos grupos (G1 e G2), bem como a estatística comparativa das variáveis categóricas (estado marital, escolaridade, raça/cor da pele, filhos, número de processos, número de processos por violência sexual, tipo de crime e proximidade com a vítima) entre os grupos.

Como seria esperado, o G1 responde por uma maior variedade de crimes com relação ao G2 $(p<0,05)$, o G2 tem mais proximidade (parentesco) com a vítima do que o G1, e o G2 é mais propenso a escolher sua própria residência como local do abuso. $\mathrm{O}$ tamanho do efeito mensurado pelo $v$ de Cramer mostrou os seguintes efeitos: associação relativamente forte entre os grupos e versatilidade criminal $(v$ Cramer $=0,446)$, proximidade com a vítima $(v$ Cramer $=0,585)$ e local do crime $(v$ Cramer=0,536).

Tabela 3

Correlação entre Idade ao Ser Preso e Pontuação por Fator (1 e 2) e Pontuação Total do PCL-R

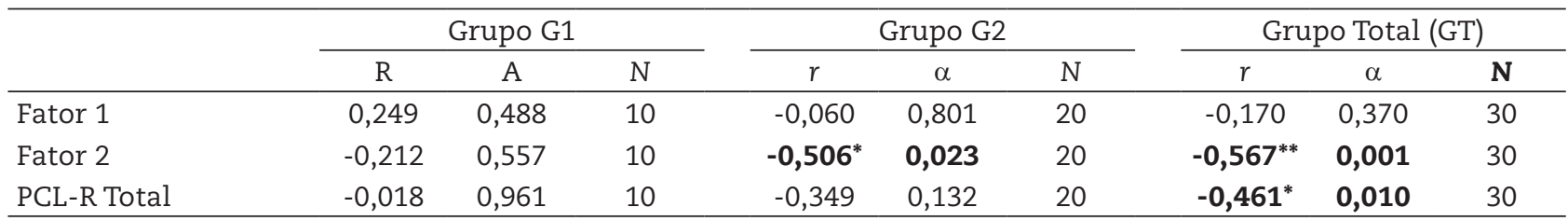

Nota. $r=$ Coeficiente de correlação de Pearson; $\alpha=$ nível de significância bicaudal; $N=$ número de indivíduos com informações nesse item; **A correlação é significativa no nível 0,01; *A correlação é significativa no nível 0,05

Tabela 4

Diferenças entre os Grupos para as Variáveis Categóricas dos Dados Sociodemográficos e do Perfil Criminal

\begin{tabular}{|c|c|c|c|c|}
\hline Variáveis & $\mathrm{G1}(\mathrm{N}=10)$ & $\mathrm{G} 2(\mathrm{~N}=20)$ & $p^{* *}$ & v Cramer \\
\hline \multicolumn{5}{|l|}{ Estado marital } \\
\hline Sem companheira & $8(80,0)$ & $10(50,0)$ & 0,235 & 0,289 \\
\hline Com companheira & $2(20,0)$ & $10(500)$ & & \\
\hline \multicolumn{5}{|l|}{ Escolaridade (anos) } \\
\hline$<4$ & $3(30,0)$ & $5(25,0)$ & 0,957 & 0,054 \\
\hline $5-8$ & $6(60,0)$ & $13(65,0)$ & & \\
\hline$>8$ & $1(10,0)$ & $2(10,0)$ & & \\
\hline
\end{tabular}


Tabela 4 (continuação) Diferenças entre os Grupos para as Variáveis Categóricas dos Dados Sociodemográficos e do Perfil Criminal

\begin{tabular}{|c|c|c|c|c|}
\hline Variáveis & $\mathrm{G1}(\mathrm{N}=10)$ & $\mathrm{G} 2(\mathrm{~N}=20)$ & $p^{* *}$ & v Cramer \\
\hline \multicolumn{5}{|l|}{ Raça/cor da pele (autodeclarada) } \\
\hline Branca & $3(37,5)$ & $10(55,6)$ & 0,545 & 0,216 \\
\hline Parda & $4(50,0)$ & $5(27,8)$ & & \\
\hline Negra & $1(12,5)$ & $3(16,7)$ & & \\
\hline \multicolumn{5}{|l|}{ Filhos } \\
\hline Não & $5(50,0)$ & $11(55,0)$ & 1,000 & 0,047 \\
\hline $\operatorname{Sim}$ & $5(50,0)$ & $9(45,0)$ & & \\
\hline \multicolumn{5}{|l|}{ Número de processos } \\
\hline 1 & $6(60,0)$ & $17(85,0)$ & 0,181 & 0,279 \\
\hline $2-5$ & $4(40,0)$ & $3(15,0)$ & & \\
\hline \multicolumn{5}{|c|}{ Número de processos por violência sexual } \\
\hline 1 & $8(80,0)$ & $18(90,0)$ & 0,584 & 0,139 \\
\hline $2-5$ & $2(20,0)$ & $2(10,0)$ & & \\
\hline \multicolumn{5}{|l|}{ Tipo de crime } \\
\hline Somente sexual & $5(50,0)$ & $18(90,0)$ & 0,026 & 0,446 \\
\hline Sexual e outro & $5(50,0)$ & $2(10,0)$ & & \\
\hline \multicolumn{5}{|l|}{ Proximidade com a vítima* } \\
\hline Parentesco & $3(30,0)$ & $17(85,0)$ & 0,006 & 0,585 \\
\hline Nenhum & $2(20,0)$ & $2(10,0)$ & & \\
\hline Amizade ou vizinhança & $5(50,0)$ & $1(5,0)$ & & \\
\hline \multicolumn{5}{|l|}{ Sexo das vítimas* } \\
\hline Masculino & $1(10,0)$ & $3(15,0)$ & 0,704 & 0,069 \\
\hline Feminino & $9(90,0)$ & $17(85,0)$ & & \\
\hline \multicolumn{5}{|l|}{ Local do crime* } \\
\hline Residência da vítima & $5(50,0)$ & $10(50,0)$ & 0,035 & 0,536 \\
\hline Residência do AVS & - & $8(40,0)$ & & \\
\hline Terreno baldio ou matagal & $2(20,0)$ & $1(5,0)$ & & \\
\hline Outro & $3(30,0)$ & $1(5,0)$ & & \\
\hline
\end{tabular}

Nota. ${ }^{*}$ Considerado a primeira vítima; ${ }^{* *}$ Qui-quadrado de Pearson para tendência ou exato de Fisher

\section{Discussão}

Tomando como base os dados coletados e analisados, o perfil mais frequente de AVS do sexo masculino contra crianças de adolescentes foi de idade entre 30 e 40 anos, em sua maioria de raça/cor da pele branca, com ensino fundamental incompleto, solteiro e trabalhador braçal (predominantemente da construção civil). Esse AVS normalmente respondia criminalmente apenas a processos sexuais, sem histórico de reincidência criminal, possuía alguma proximidade com a vítima, geralmente eram pais ou padrastos, e tinham preferência por vítimas crianças e adolescentes. Embora os AVSs contra crianças e adolescentes componham um grupo heterogêneo, provenientes de backgrounds diferentes, o perfil encontrado corroborou com os dados encontrados na literatura científica sobre o tema (Huss, 2011; Martins \& Jorge, 2010; Santos et al., 2015; Serafim et al., 2009; Soares et al., 2016). É importante destacar que quaisquer conclusões gerais sobre a associação entre psicopatia e dados sociodemográficos devem aguardar por resultados de estudos epidemiológicos em larga escala.

Os dados apontam que um terço dos AVSs contra crianças e adolescentes $(N=10 ; 33,3 \%)$ preenchiam os critérios para criminosos com psicopatia (conforme o critério de pontuação maior ou igual a 30 no PCL-R). A incidência de psicopatia em AVS, encontrada neste estudo, é ligeiramente maior do que aquela encontrada em um estudo realizado por Young et al. (2010), em que a incidência foi de $28 \%$ dos AVSs (PCL-R $>30$ ). Essa porcentagem se aproxima das descritas na literatura, onde encontra-se que a psicopatia está presente em $15 \%$ a $20 \%$ da população carcerária no geral e se torna ligeiramente maior quando se trata de AVS (Hare, 2003; Hare et al., 1991; Hauck Filho et al., 2012; Hemphill et al., 1998).

Pode-se observar diferenças significativas entre os grupos G1 (considerados psicopatas) e G2 (considerados não psicopatas), com relação a algumas variáveis coletadas por meio dos processos que estavam disponíveis no cartório do presídio. O grupo de participantes 
considerados psicopatas prejudicou um número significativamente maior de vítimas do que os participantes considerados não psicopatas. Além disso, há significativamente mais registros de fugas, rebeliões, versatilidade criminal, maior o número de processos de modo geral e de processos por crimes sexuais, o que, consequentemente, faz com que o tempo total de pena seja também expressivamente maior no G1.

Tais observações em relação ao G1 apontam que esse grupo foi composto por indivíduos mais indisciplinados no sistema carcerário e com mais chances de reincidirem criminalmente (Balsis et al., 2017; Gacono et al., 2008). Além disso, a literatura aponta que essas pessoas apresentam respostas não satisfatórias nos programas de reabilitação (Gacono et al., 2008; Hare, 2006; Hill et al., 2008; Morana, 2003).

Quanto ao baixo índice de reincidência criminal observado nos dois grupos (G1=0,7 e G2=0,3), essa é uma questão muito delicada. Serafim, Saffi et al. (2009) destacam que mais da metade dos criminosos que acabaram de cumprir pena voltam para a penitenciária com menos de um ano. Esse percentual sobe para 77,9\% em dois anos. Assim, a taxa de reincidência varia de $18 \%$ a $45 \%$. Porém, Prentky et al. (2008) alertam sobre a pouca confiabilidade dessa informação, ressaltando que a reincidência criminal é altamente variável, o que exige cautela na hora de apresentar quaisquer conclusões sobre a reincidência de AVS. Além disso, o típico agressor se engaja em vários comportamentos pervertidos e molesta uma média de 117 jovens, cuja maioria não presta queixa (Serafim et al., 2009), o que torna difícil mensurar esse dado. Embora os resultados alcançados por Langton et al. (2006), Olver e Wong (2009), Hawes et al. (2013) apontem a disposição para maior reincidência criminal em examinandos considerados psicopatas (PCL-R > 30), os dados encontrados no presente estudo não sugerem tal fato. Isso pode ter ocorrido pelo fato de não haver histórico de reincidência criminal nos processos coletados no cartório, o que não significa que os AVSs participantes da pesquisa não tenham reincidido. Tais achados vão ao encontro dos resultados alcançados por Olver e Wong (2009), em que não houve um aumento significativo de reincidência para os AVSs e psicopatia.

Os resultados não apontam diferenças significativas entre o G1 e G2 para vítima criança, ou seja, AVSs com mais traços de psicopatia não abusam mais de crianças do que os AVSs com menos traços. Por outro lado, os AVSs psicopatas revelaram preferência por vítimas adolescentes e adultas, respaldando os resultados encontrados por Yesuron (2015), apontando que o AVS psicopata tem como vítima de seu crime sexual uma pessoa maior de idade, indicando um tipo de violência sexual com adultos, caracterizada pela intimidação e uso da força.

Os AVSs do G1 eram significativamente mais novos que os AVSs do G2. Tal achado assemelha-se ao achado do estudo realizado por Young et al. (2010), que apontou que AVSs com mais traços de psicopatia (PCL-R > 30) são mais jovens que os AVSs com menos traços. Embora a literatura trace o indivíduo psicopata como o indivíduo que inicia a vida criminosa muito cedo, esse dado não pôde ser observado neste estudo por conta da ausência de histórico criminal dos AVSs na adolescência, uma vez que os processos no cartório não remetem a nenhuma passagem destes por programas socioeducativos, o que acabou dificultando o preenchimento desse item no PCL-R, e isso pode ter contribuído para a não pontuação nesse tópico. Além disso, psicopatas tendem a ser menos impulsivos após os 40 ou 50 anos. Huss e Langhinrichsen-Rohling (2000) e Huss (2011) apontam que os psicopatas apresentam um decréscimo considerável em seu comportamento criminal após os 40 anos de idade.

É provável que o G2 seja composto por vários AVSs situacionais, que são oportunistas e impulsivos, que avaliam as características gerais da vítima e usam como critérios de escolha a disponibilidade da vítima e a oportunidade apresentada. Não possuem nenhum transtorno psiquiátrico e podem ser pessoas que foram expostas a situações de estresse extremo. Geralmente, são pessoas que possuem poder absoluto sobre a vítima e têm dificuldade no controle dos impulsos (Holmes \& Holmes, 2002; Serafim et al., 2009). Outra possibilidade é que esse G2 também seja composto por vários pedófilos molestadores, que têm um padrão de comportamento mais invasivo e agressivo. São inteligentes e agem de forma premeditada. Sabem que estão agindo fora da lei, porém, intelectualizam seu comportamento, convencendo-se de que não estão cometendo nenhum crime e que seu comportamento é socialmente aceitável. É possível também que nesse grupo também tenha AVS com algum grau de retardo mental ou com traços psicóticos, que está impossibilitado de discernir comportamentos adequados ou não com relação à sua prática sexual.

Por fim, vale ressaltar que este estudo possui como uma de suas limitações a utilização de uma perspectiva categorial para a identificação da psicopatia e utilização de um ponto de corte para sua identificação. Embora esta tenha sido uma proposta bastante aceita, novos estudos têm suportado uma estrutura dimensional para esse e outros transtornos de personalidade. Dessa forma, sugere-se que novos estudos considerem essa nova perspectiva nas análises de dados (Carvalho et al., 2019; Hauck Filho et al., 2009).

\section{Agradecimentos}

Não há menções.

\section{Financiamento}

Todas as fontes de financiamento para elaboração e produção do estudo (coleta, análise e interpretação dos dados, bem como, escrita dos resultados no presente no manuscrito) foram fornecidas pelo projeto de pesquisa 
'código do financiamento 201610267000603 e Fundação de Amparo à Pesquisa do Estado de Goiás.

\section{Contribuições dos autores}

Declaramos que todos os autores participaram da elaboração do manuscrito. Especificamente, as autoras Julia Nunes Souza Teixeira e Ana Cristina Resende participaram da redação inicial do estudo - conceitualização, investigação, visualização, os autores Julia Nunes Souza Teixeira, Ana Cristina Resende e Rodrigo Perissinotto participaram da análise dos dados, e as autoras Julia Nunes Souza Teixeira e Ana Cristina Resende participaram da redação final do trabalho - revisão e edição.

\section{Disponibilidade dos dados e materiais}

Todos os dados e sintaxes gerados e analisados durante esta pesquisa serão tratados com total sigilo devido às exigências do Comitê de Ética em Pesquisa em Seres Humanos. Porém, o conjunto de dados e sintaxes que apoiam as conclusões deste artigo estão disponíveis mediante razoável solicitação ao autor principal do estudo.

\section{Conflito de interesses}

Os autores declaram que não há conflitos de interesses.

\section{Referências}

Balsis, S., Busch, A. J., Wilfong, K. M., Newman, J. W., \& Edens. J. F. (2017). A statistical consideration regarding the threshold of the psychopathy checklist-revised. Journal of Personality Assessment, 95(5), 494-502. https://doi.org/10.1080/00223891.2017.1281819

Blais, J. (2015). Preventative detention decisions: Reliance on expert assessments and evidence of partisan allegiance within the Canadian context. Behavioral Sciences \& the Law, 33(1), 74-91. https://doi.org/10.1002/bsl.2155

Carvalho, L. F., Hauck Filho, N., Pianowski, G., \& Muner, L. C. (2019). Latent structure of antisocial and borderline personality disorders: A taxometric research. Paidéia (Ribeirão Preto), 29, e2902, 1-10. https://doi.org/10.1590/1982-4327e2902

Cicchetti, D. V. (1994). Guidelines, criteria, and rules of thumb for evaluating normed and standardized assessment instruments in psychology. Psychological Assessment, 6(4), 284. https://doi.org/10.1037/1040-3590.6.4.284

Cohen, J. (1988). Statistical Power Analysis for the Behavioral Sciences (2nd ed.). Hillsdale, NJ: Lawrence Erlbaum Associates, Publishers.

Cooke, D. J. \& Michie, C. (2001). Refining the construct of psychopathy: towards a hierarchical model. Psychological Assessment, 13(2), 171188. https://doi.org/10.1037/1040-3590.13.2.171

DeMatteo, D., Edens, J. F., Galloway, M., Cox, J., Smith, S. T., Koller, J. P., \& Bersoff, B. (2014). Investigating the role of the Psychopathy Check- list-Revised in United States case law. Psychology, Public Policy, and Law, 20(1), 96-107. https://doi.org/10.1037/a0035452

D’Silva, K., Duggan, C., \& McCarthy, L. (2004). Does treatment really make psychopaths worse? A review of the evidence. Journal of Personality Disorders, 18(2), 163-177. https://doi.org/10.1521/pedi.18.2.163.32775

Frick, P. J., \& Marsee, M. A. (2018). Psychopathy and developmental pathways to antisocial behavior in youth. Em C. J. Patrick (Ed.), Handbook of psychopathy (pp. 456-477). New York: Guilford.

Gacono, V. B., Meloy, J. R., \& Bridges, M. (2008). A Rorschach understanding of psychopats, sexual homicide perpetrators, and nonviolent pedophiles. Em C. Gacono \& B. Evans. The Handbook of Forensic Rorschach Assessment (pp. 379-402) New York, Routledge.

Guy, L. S., Kusaj, C., Packer, I. K., \& Douglas, K. S. (2015). Influence of the HCR-20, LS/CMI, and PCL-R on decisions about parole suitability among lifers. Law and Human Behavior, 39(3), 232-243. https://doi.org/10.1037/ lhb0000111

Hare, R. D., \& Neumann, C. S. (2006). The PCL-R assessment of Psychopathy: Development, structural properteis and newdirections. Em C. Patrick (Ed.), Handbook of Psychopathy (pp. 58-90). New York: Guilford.

Hare, R. D. (1991). The Hare Psychopathy Checklist-Revised (PCL-R). Toronto, Ontario: Multi-Health Systems.

Hare, R. D. (1995). Psychopaths: New Trends in Research. Harvard Mental Health Letter, 12, 4-5. Recuperado de http://minet.org/www. trancenet.net/hearts/research/psychopath.shtml.

Hare, R. D. (2003). Hare Psychopathy Checklist-Revised technical manual (2nd ed.). Toronto, ON, Canada: Multi-Health Systems.

Hare, R. D. (2006). Psychopathy: A clinical and forensic overview. Psychiatric Clinic of North American, 29(3), 709-724. https://doi.org/10.1016/j. psc.2006.04.007.

Hare, R. D., Hart, S. D., \& Harpur, T. J. (1991). Psychopathy and the DSM-IV criteria for antisocial personality disorder. Journal of Abnormal Psychology, 100(3), 391. Recuperado de https://psycnet.apa.org/record/1991-33364-001

Hauck Filho, N., Teixeira, M. A. P., \& Dias, A. C. G. (2009). Psicopatia: O construto e sua Avaliação. Avaliação Psicológica, 8(3), $337-346$. Recuperado de http://pepsic.bvsalud.org/scielo.php?script=sci_arttext\&pid=S1677-04712009000300006

Hauck Filho, N., Teixeira, M. A. P., \& Dias, A. C. G. (2012). Psicopatia: Uma perspectiva dimensional e não-criminosa do construto. Avances en Psicología Latinoamericana, 30(2), 317-372. Recuperado de https://dialnet.unirioja.es/servlet/articulo?codigo=4456911

Hawes, S. W., Boccaccini, M. T., \& Murrie, D. C. (2013). Psychopathy and the combination of psychopathy and sexual deviance as predictors of sexual recidivism: Meta-analytic findings using the Psychopathy Checklist-Revised. Psychological Assessment, 25(1), 233-243. https:// doi.org/10.1037/a0030391

Hemphill, J. F., Hare, R. D., \& Wong, S. (1998). Psychopathy and recidivism: A review. Legal and Criminological Psychology, 3(1), 139-170. Recuperado de https://onlinelibrary.wiley.com/doi/abs/10.1111/j.2044-8333.1998.tb00355.x

Hill, A., Habermann, N., Klusmann, D., Berner, W., \& Briken, P. (2008). Criminal recidivism in sexual homicide perpetrators. International Journal of Offender Therapy and Comparative Criminology, 52(1), 5-20. https://doi.org/10.1177/0306624X07307450

Holmes, R. M., \& Holmes, S. T. (2002). Profiling violent crimes: An investigative tool. Neu Delhi: US

Hunsley, J., \& Mash, E. J. (2008). A Guide to Assessments that Work. New York, NY: Oxford University Press.

Huss, M. T. (2011). Psicologia forense: Pesquisa, prática clínica e aplicações. Porto Alegre: Artmed.

Huss, M. T., \& Langhinrichsen-Rohling, J. (2000). Identification of the psychopathic batterer: The clinical, legal, and policy implications. Aggression and Violent Behavior, 5(4), 403-422. https://doi.org/10.1016/S1359-1789(98)00038-X 
Krstic, S., Neumann, C. S., Roy, S., Robertson, C. A., Knight, R. A., \& Hare, R. D. (2017). Using latent variable - And person-centered approaches to examine the role of psychopathic traits in sex offenders. Personality Disorders, 8(3), 207-216 https://doi.org/10.1037/ per0000249

Langton, C. M., Barbaree, H. E., Harkins, L., \& Peacock, E. J. (2006). Sex offenders' response to treatment and its association with recidivism as a function of psychopathy. Sexual Abuse: A Journal of Research and Treatment, 18(1), 99-120. https://doi.org/10.1177/107906320601800107

Lu, Y., Freeman, N. J., \& Sandler, J. C. (2015). Predictors of the sex offender civil commitment trial outcomes in New York State. Law and Human Behavior, 39(5), 514-524. https://doi.org/10/1037lhb0000143

Martins, C. B. G., \& Jorge, M. H. P. M. (2010). Abuso sexual na infância e adolescência: Perfil das vítimas e agressores em município do sul do Brasil. Texto Contexto Enfermagem, 19(2), 246-255. https://doi.org/10 1590/S0104-07072010000200005

Misaka, M. Y. (2014). Violência sexual infantil intrafamiliar: Não há apenas uma vítima. Revista Direitos Sociais e Políticas Públicas (UNIFAFIBE), 2(2), 237-277. https://doi.org/org/1025245/rdspp.v2i2.39

Mokros, A., Vohs, K., \& Habermeyer, E. (2014). Psychopathy and violent reoffending in German-speaking countries. European Journal of Psychological Assessment, 30, 117-129. https://doi.org/10.1028/1015-5759

Morana, H. (2004). Escala Hare PCL-R: Critérios para pontuação de psicopatia revisados. Versão brasileira. São Paulo: Casa do Psicólogo.

Morana, H. C. P. (2003). Identificação do ponto de corte para a escalo PCL-R (Psychopathy Checklist Revised) em população forense brasileira: Caracterização de dois subtipos de personalidade; Transtorno global e parcial [Tese de doutorado não publicada, Faculdade de Medicina da Universidade de São Paulo].

Neumann, C. S., \& Hare, R. D. (2008). Psychopathic traits in a large community sample: Links to violence, alcohol use, and intelligence. Journal of Consulting and Clinical Psychology, 76(5), 893-899. https://doi.org/10.1037/0022-006X.76.5.893.

Olver, M. E., \& Wong, S. C. P. (2009). Therapeutic responses of psychopathic sexual offenders: treatment attrition, therapeutic change, and long-term recidivism. Journal of Consulting and Clinical Psychology, 77(2), 328-336. https://doi.org/10.1037/a0015001

Prentky, R. A., Knight, R. A., \& Lee, A. F. S. (2008). Child sexual molestation. Em C. R. Bartol \& A. M. Bartol. Current perspectives in forensic psychology and criminal behavior (p. 123-134). Los Angeles: Sage.

Rea, L. M., \& Parker, R. A. (1992). Designing and conducting survey research. San Francisco: Jossey-Boss.

Riquelme, C. A., Pérez, N., \& Muñoz, C. G. (2004). Adaptación de la Escala de Calificación de la Psicopatía Revisada (PCL-R) de Robert Hare en población reclusa del Centro de Detención Preventiva de San Miguel. [Trabalho de Conclusão de Curso, Graduação - Facultad de Ciencias Sociales, Universidade do Chile]. Recuperado de http://repositorio.uchile.cl/handle/2250/106385

Santos, C. A., Costa, M. C. O., Amaral, M. T. R., Nascimento Sobrinho, C. L., Musse, J. O., \& Costa, A. M. (2015). Agressor sexual de crianças e adolescentes: Análise de situações relacionadas à violação e vítimas. Adolescência e Saúde, 12(3), 7-20. Recuperado de http:// www.adolescenciaesaude.com/detalhe_artigo.asp?id $=519$

Serafim, A. D. P., Saffi, F., Rigonatti, S. P., Casoy, I., \& Barros, D. M. D. (2009). Perfil psicológico e comportamental de agressores sexuais de crianças. Revista de Psiquiatria Clínica, 36(3), 101-111. https://doi.org/10.1590/S0101-60832009000300004

Sharma, B. R. (2003). Disorders of sexual preference and medicolegal issues thereof. American Journal of Forensic Medicine Pathology, 24(3), 277-282. https://doi.org/10.1097/01.paf.0000069503.21112.d2

Shrout, P. E., \& Fleiss, J. L. (1979). Intraclass correlations: Uses in assessing rater reliability. Psychological Bulletin, 86(2), 420. Recuperado de https://psycnet.apa.org/record/1979-25169-001

Singh, J. P., Grann, M., \& Fazel, S. (2011). A comparative study of violence risk assessment tools: A systematic review and metaregression analysis of 68 studies involving 25,980 participants. Clinical Psychology Review, 31(3), 499-513. https://doi.org/10.1016/j.cpr.2010.11.009

Soares, E. M. R., Silva, N. L., Matos, M. A. S., Araújo, E. T. H., Silva, L. R., \& Lago, E. C. (2016). Perfil da violência sexual contra crianças e adolescentes. Revista Interdisciplinar, 9(1), 87-96. https://doi.org/10.1590/S1413-81232007000500010

Valverde, C. S. (2005). Psicopatía y violación: Um estúdio com ofensores sexuales costarricenses. Medicina Legal de Costa Rica, $22(1)$, 1-11. Recuperado de https://pesquisa.bvsalud.org/portal/resource/pt/lil-432867

Verschuere, B., Grothe, A. S. v. G., Waldorp, L., Watts, A. L., Edens, J. F., Skeem, J., \& Noordhof, A. (2017). What Features of Psychopathy Might be Central? A Network Analysis of the Psychopathy Checklist-Revised (PCL-R) in Three Large Samples. https://doi.org/10.31219/osf.io/95ewr

Yesuron, M. R. (2015). Perfil psicopatológico de delincuentes sexuales. Anuario de Investigaciones de la Facultad de Psicología, 2(1), $192-203$. Recuperado de https://revistas.unc.edu.ar/index.php/aifp/article/view/13178/13375

Young, M. H., Justice, J. V., \& Edberg, P. (2010). Sexual offenders in prison psychiatric treatment: A biopsychosocial description. International Journal of Offender Therapy and Comparative Criminology, 54(1), 92-112. https://doi.org/10.1177/0306624X08322373

recebido em fevereiro de 2019 aprovado em novembro de 2020

\section{Sobre os autores}

Julia Nunes de Souza Teixeira é psicóloga (PUC Goiás), doutoranda do programa de pós-graduação em Psicologia Social (UERJ), Mestre em Psicologia (PUC Goiás), especialista em Psicologia Organizacional (UNIARA) e em Gestão Estratégica de Pessoas e Psicologia Organizacional pela Universidade Metodista de São Paulo.

Ana Cristina Resende é psicóloga (PUC Goiás) é doutora em Psicologia pela Universidade Federal do Rio Grande do Sul (UFRGS) e, atualmente, professora no Programa de Pós-Graduação Stricto Sensu em Psicologia da Pontifícia Universidade Católica de Goiás, Goiânia.

Rodrigo Perissinotto é psicólogo (PUC Goiás), doutorando e mestre em Psicologia pela PUC Goiás e, atualmente, é professor de graduação na Unidade de Biociências no Centro Universitário de Mineiros (UNIFIMES).

\section{Como citar este artigo}

Teixeira, J. N. S., Resende, A. C., \& Perissinotto, R. (2021). Psicopatia e Autores de Violência Sexual contra Crianças e Adolescentes. Avaliação Psicológica, 20(1), 111-121. http://dx.doi.org/10.15689/ap. 2021.2001.17724.12 